\title{
A microscopic quantum dynamics approach to the dilute condensed Bose gas
}

\author{
Thorsten Köhler and Keith Burnett \\ Clarendon Laboratory, Department of Physics, \\ University of Oxford, Oxford, OX1 3PU, United Kingdom
}

(November 3, 2018)

We derive quantum evolution equations for the dynamics of dilute condensed Bose gases. The approach contains, at different orders of approximation, for cases close to equilibrium, the Gross Pitaevskii equation and the first order Hartree Fock Bogoliubov theory. The proposed approach is also suited for the description of the dynamics of condensed gases which are far away from equilibrium. As an example the scattering of two Bose condensates is discussed.

PACS numbers: 03.75.Fi, 34.50.-s

\section{INTRODUCTION}

The description of the evolution of condensates far from equilibrium is now a matter of considerable importance to the field of matter wave physics. This includes colliding [1] and collapsing [2,3] condensates where components of the overall condensed system are moving rapidly with respect to one another. Numerous quantitative theoretical studies of partially condensed gases to date have relied mostly on the time dependent GrossPitaevskii approach and extended kinetic theories (see, e.g., [4 [6]) that are appropriate to fluctuations and excitations around thermal equilibrium situations. These general approaches all depend on the contact potential approximation of the binary interactions which restricts two body collisions to zero momenta. New methods are, therefore, required to describe systems outside this restricted domain.

In this paper we shall describe a technique that we believe can provide a comprehensive approach to the description of evolving condensates. We use the method of non-commutative cumulants [7] to derive a set of coupled equations that should provide an accurate description in regimes where previous approaches fail. We shall explain the formal basis of the method before applying it to the partially condensed gas. We shall then focus on the specific case of a colliding pair of condensates to show the utility of the method. The general coupled equations should also be useful for the description of fourwave mixing experiments, for collapsing condensates and other cases where bulk relative motion of condensates is observed. In the next section we shall define the Hamiltonian for the systems we wish to study and find the formal equations of motion for the correlation functions.

\section{QUANTUM EVOLUTION EQUATIONS FOR CORRELATION FUNCTIONS}

A many body system of identical boson atoms with a pair interaction $V(\mathbf{r})$ is described by the Hamiltonian

$$
\begin{aligned}
& H=\int d \mathbf{x} \psi^{\dagger}(\mathbf{x}) H_{1 \mathrm{~B}}(\mathbf{x}) \psi(\mathbf{x}) \\
& +\frac{1}{2} \int d \mathbf{x}_{1} \int d \mathbf{x}_{2} \psi^{\dagger}\left(\mathbf{x}_{1}\right) \psi^{\dagger}\left(\mathbf{x}_{2}\right) V\left(\mathbf{x}_{1}-\mathbf{x}_{2}\right) \psi\left(\mathbf{x}_{2}\right) \psi\left(\mathbf{x}_{1}\right) .
\end{aligned}
$$

Here $H_{1 \mathrm{~B}}(\mathbf{x})$ is the one body Hamiltonian containing the kinetic energy $-\hbar^{2} \Delta_{\mathbf{x}} / 2 m$ and the trapping potential $V_{\text {trap }}(\mathbf{x})$. The field operators satisfy the boson commutation relations

$$
\begin{aligned}
{\left[\psi\left(\mathbf{x}_{1}\right), \psi\left(\mathbf{x}_{2}\right)\right] } & =0, \\
{\left[\psi\left(\mathbf{x}_{1}\right), \psi^{\dagger}\left(\mathbf{x}_{2}\right)\right] } & =\delta\left(\mathbf{x}_{1}-\mathbf{x}_{2}\right) .
\end{aligned}
$$

Throughout this article the state of the many body system at time $t$ is described by a statistical operator $\rho(t)$ and the expectation value of an arbitrary operator $A$ is denoted by

$$
\langle A\rangle_{t}=\operatorname{Tr}(\rho(t) A) .
$$

The Schrödinger equation then yields the dynamic equation

$$
i \hbar \frac{\partial}{\partial t}\langle A\rangle_{t}=\langle[A, H]\rangle_{t}
$$

In the following, expectation values in Eq. (3) are denoted as correlation functions if $A$ is a product of creation or annihilation operators. Since every expectation value can be expanded in linear combinations of normal ordered correlation functions their dynamics determines all physical properties of the many body system. According to Eq. (雨) the system of dynamic equations for the normal ordered correlation functions up to the order of $n$ reads

$$
\begin{aligned}
& i \hbar \frac{\partial}{\partial t}\left\langle\psi\left(\mathbf{x}_{1}\right)\right\rangle_{t}=\left\langle\left[\psi\left(\mathbf{x}_{1}\right), H\right]\right\rangle_{t}, \\
& i \hbar \frac{\partial}{\partial t}\left\langle\psi\left(\mathbf{x}_{2}\right) \psi\left(\mathbf{x}_{1}\right)\right\rangle_{t}=\left\langle\left[\psi\left(\mathbf{x}_{2}\right) \psi\left(\mathbf{x}_{1}\right), H\right]\right\rangle_{t}, \\
& i \hbar \frac{\partial}{\partial t}\left\langle\psi^{\dagger}\left(\mathbf{x}_{2}\right) \psi\left(\mathbf{x}_{1}\right)\right\rangle_{t}=\left\langle\left[\psi^{\dagger}\left(\mathbf{x}_{2}\right) \psi\left(\mathbf{x}_{1}\right), H\right]\right\rangle_{t}, \\
& \vdots \\
& i \hbar \frac{\partial}{\partial t}\left\langle\psi^{\dagger}\left(\mathbf{x}_{n}\right) \cdots \psi\left(\mathbf{x}_{1}\right)\right\rangle_{t}=\left\langle\left[\psi^{\dagger}\left(\mathbf{x}_{n}\right) \cdots \psi\left(\mathbf{x}_{1}\right), H\right]\right\rangle_{t} .
\end{aligned}
$$


The system Eq. (5) is incomplete since the commutator of a product of $n$ field operators with $H$ contains products of $n+2$ field operators. The main problem thus consists in truncating Eq. (5) appropriately. An approximate method to close the kinetic equations at an arbitrary order has been proposed by Fricke [7 in order to describe the dynamics of many body systems on short time scales. In the latter approach Eq. (5) is transformed into an equivalent system of differential equations for what are termed non-commutative cumulants. This article contains the extension of Fricke's work to the description of the dynamics of dilute Bose gases and their equilibrium properties.

\section{DYNAMIC EQUATIONS FOR NON COMMUTATIVE CUMULANTS}

Given a set of boson creation and annihilation operators $B_{1}, B_{2}, \ldots$ the cumulant expansion of their respective correlation functions is defined recursively by []

$$
\begin{aligned}
& \left\langle B_{1}\right\rangle=\left\langle B_{1}\right\rangle^{c} \\
& \left\langle B_{1} B_{2}\right\rangle=\left\langle B_{1} B_{2}\right\rangle^{c}+\left\langle B_{1}\right\rangle^{c}\left\langle B_{2}\right\rangle^{c} \\
& \left\langle B_{1} B_{2} B_{3}\right\rangle=\left\langle B_{1} B_{2} B_{3}\right\rangle^{c}+\left\langle B_{1}\right\rangle^{c}\left\langle B_{2} B_{3}\right\rangle^{c} \\
& \quad+\left\langle B_{2}\right\rangle^{c}\left\langle B_{1} B_{3}\right\rangle^{c}+\left\langle B_{3}\right\rangle^{c}\left\langle B_{1} B_{2}\right\rangle^{c}+\left\langle B_{1}\right\rangle^{c}\left\langle B_{2}\right\rangle^{c}\left\langle B_{3}\right\rangle^{c},
\end{aligned}
$$$$
\vdots
$$

The expressions $\left\langle B_{1} \ldots \cdot B_{n}\right\rangle^{c}$ define the non commutative cumulants we shall use. The cumulants can be obtained directly in terms of correlation functions through

$$
\begin{aligned}
& \left\langle B_{1} \cdots B_{n}\right\rangle^{c} \\
& =\left.\frac{\partial}{\partial x_{1}} \cdots \frac{\partial}{\partial x_{n}} \log \left\langle e^{x_{1} B_{1}} \cdots e^{x_{n} B_{n}}\right\rangle\right|_{x_{1}=0, \ldots, x_{n}=0} .
\end{aligned}
$$

In contrast to their respective correlation functions the cumulants should decrease with increasing order, as long as the system is not too far away from the interaction free equilibrium. More precisely, for an ideal Bose gas in thermal equilibrium all cumulants containing more than two field operators vanish. In fact, if the state of an ideal gas is given by the grand canonical density matrix, according to Wick's theorem of statistical mechanics [8], every number conserving normal ordered correlation function $\left\langle B_{1} \cdots B_{n}\right\rangle$ splits into a sum of all possible products of contractions $\left\langle B_{i} B_{j}\right\rangle$ which preserve the initial order of the operators. Since the expectation value of a single field operator vanishes, the contractions equal their respective second order cumulants. Inserting the Wick decompositions of the correlation functions into the left hand sides of Eqs. (6) shows successively that only the number conserving cumulants of the second order can be different from zero. In this sense the higher order cumulants are a measure of the deviations of the physical system from the interaction free equilibrium.
Among other general properties it is worth mentioning that in the absence of a pair interaction the cumulants satisfy the same dynamic equations as their respective correlation functions. In addition, all field operators in $\left\langle B_{1} \cdots B_{n}\right\rangle^{c}$ commute as soon as the order $n$ exceeds two, i.e.

$$
\left\langle\cdots B_{i} B_{i+1} \cdots\right\rangle^{c}=\left\langle\cdots B_{i+1} B_{i} \cdots\right\rangle^{c} .
$$

According to Eq. (7) the cumulants of the order of $n>1$ depend only on the "deviation operators" $B_{i}-\left\langle B_{i}\right\rangle$, i.e.

$$
\left\langle B_{1} \cdots B_{n}\right\rangle^{c}=\left\langle\left(B_{1}-\left\langle B_{1}\right\rangle\right) \cdots\left(B_{n}-\left\langle B_{n}\right\rangle\right)\right\rangle^{c} .
$$

Hence, the cumulant expansion Eq. (6) is also useful for the description of Bose condensed systems with non number conserving correlation functions.

Due to their particular roles in the description of condensed dilute Bose gases throughout this article, the first and second order normal ordered cumulants are denoted as follows:

$$
\begin{aligned}
\Psi(\mathbf{x}, t) & =\langle\psi(\mathbf{x})\rangle_{t}^{c} \\
\Phi\left(\mathbf{x}_{1}, \mathbf{x}_{2}, t\right) & =\left\langle\psi\left(\mathbf{x}_{2}\right) \psi\left(\mathbf{x}_{1}\right)\right\rangle_{t}^{c} \\
\Gamma\left(\mathbf{x}_{1}, \mathbf{x}_{2}, t\right) & =\left\langle\psi^{\dagger}\left(\mathbf{x}_{2}\right) \psi\left(\mathbf{x}_{1}\right)\right\rangle_{t}^{c}
\end{aligned}
$$

The first order cumulant $\Psi(\mathbf{x}, t)$ is usually referred to as the condensate wave function and $\Phi\left(\mathbf{x}_{1}, \mathbf{x}_{2}, t\right)$ as the pair function. $\Gamma\left(\mathbf{x}_{1}, \mathbf{x}_{2}, t\right)$ is interpreted as the one body density matrix of the non condensed fraction. The total one body correlation function then reads

$$
\begin{aligned}
\rho^{(1)}\left(\mathbf{x}_{1}, \mathbf{x}_{2}, t\right) & =\left\langle\psi^{\dagger}\left(\mathbf{x}_{2}\right) \psi\left(\mathbf{x}_{1}\right)\right\rangle_{t} \\
& =\Gamma\left(\mathbf{x}_{1}, \mathbf{x}_{2}, t\right)+\Psi\left(\mathbf{x}_{1}, t\right) \Psi^{*}\left(\mathbf{x}_{2}, t\right) .
\end{aligned}
$$

Hence, the density of the gas at the position $\mathbf{x}$ and time $t$ is given by $\rho^{(1)}(\mathbf{x}, \mathbf{x}, t)$.

The dynamic equations for the normal ordered cumulants can be derived from Eqs. (5) by commuting successively all creation operators in the commutators on the right hand sides to the left and expanding the correlation functions on both sides according to Eq. (6). Hence, the exact dynamics of the condensate wave function is described by

$$
\begin{aligned}
& i \hbar \frac{\partial}{\partial t} \Psi(\mathbf{x}, t)=H_{1 \mathrm{~B}}(\mathbf{x}) \Psi(\mathbf{x}, t) \\
& +\int d \mathbf{y} V(\mathbf{x}-\mathbf{y})\left[\left\langle\psi^{\dagger}(\mathbf{y}) \psi(\mathbf{y}) \psi(\mathbf{x})\right\rangle_{t}^{c}+\Psi(\mathbf{y}, t) \Gamma(\mathbf{x}, \mathbf{y}, t)\right. \\
& \quad+\Psi(\mathbf{x}, t) \Gamma(\mathbf{y}, \mathbf{y}, t)] \\
& +\int d \mathbf{y} V(\mathbf{x}-\mathbf{y}) \Psi^{*}(\mathbf{y}, t)[\Phi(\mathbf{x}, \mathbf{y}, t)+\Psi(\mathbf{x}, t) \Psi(\mathbf{y}, t)]
\end{aligned}
$$

In the same way the exact equations of motion for the 
pair function

$$
\begin{aligned}
& i \hbar \frac{\partial}{\partial t} \Phi\left(\mathbf{x}_{1}, \mathbf{x}_{2}, t\right)=H_{2 \mathrm{~B}}\left(\mathbf{x}_{1}, \mathbf{x}_{2}\right) \Phi\left(\mathbf{x}_{1}, \mathbf{x}_{2}, t\right) \\
& +V\left(\mathbf{x}_{1}-\mathbf{x}_{2}\right) \Psi\left(\mathbf{x}_{1}, t\right) \Psi\left(\mathbf{x}_{2}, t\right) \\
& +\left\{\int d \mathbf { y } V ( \mathbf { x } _ { 1 } - \mathbf { y } ) \left[\left\langle\psi^{\dagger}(\mathbf{y}) \psi(\mathbf{y}) \psi\left(\mathbf{x}_{2}\right) \psi\left(\mathbf{x}_{1}\right)\right\rangle_{t}^{c}\right.\right. \\
& \quad+\Psi(\mathbf{y}, t)\left\langle\psi^{\dagger}(\mathbf{y}) \psi\left(\mathbf{x}_{2}\right) \psi\left(\mathbf{x}_{1}\right)\right\rangle_{t}^{c} \\
& \quad+\Psi\left(\mathbf{x}_{1}, t\right)\left\langle\psi^{\dagger}(\mathbf{y}) \psi(\mathbf{y}) \psi\left(\mathbf{x}_{2}\right)\right\rangle_{t}^{c} \\
& \left.\quad+\Gamma(\mathbf{y}, \mathbf{y}, t) \Phi\left(\mathbf{x}_{1}, \mathbf{x}_{2}, t\right)+\Gamma\left(\mathbf{x}_{1}, \mathbf{y}, t\right) \Phi\left(\mathbf{x}_{2}, \mathbf{y}, t\right)\right] \\
& +\int d \mathbf{y} V\left(\mathbf{x}_{1}-\mathbf{y}\right) \Psi^{*}(\mathbf{y}, t)\left[\left\langle\psi(\mathbf{y}) \psi\left(\mathbf{x}_{2}\right) \psi\left(\mathbf{x}_{1}\right)\right\rangle_{t}^{c}\right. \\
& \left.\quad+\Psi(\mathbf{y}, t) \Phi\left(\mathbf{x}_{1}, \mathbf{x}_{2}, t\right)+\Psi\left(\mathbf{x}_{1}, t\right) \Phi\left(\mathbf{x}_{2}, \mathbf{y}, t\right)\right] \\
& \quad+d \mathbf{y} V\left(\mathbf{x}_{1}-\mathbf{y}\right) \Gamma\left(\mathbf{x}_{2}, \mathbf{y}, t\right) \\
& \left.\quad \quad \int\left[\Phi\left(\mathbf{x}_{1}, \mathbf{y}, t\right)+\Psi\left(\mathbf{x}_{1}, t\right) \Psi(\mathbf{y}, t)\right]\right\} \\
& \left.\quad \quad \times \quad \mathbf{x}_{1} \leftrightarrow \mathbf{x}_{2}\right\}
\end{aligned}
$$

and the density matrix of the non condensed fraction

$$
\begin{aligned}
& i \hbar \frac{\partial}{\partial t} \Gamma\left(\mathbf{x}_{1}, \mathbf{x}_{2}, t\right)=\left[H_{1 \mathrm{~B}}\left(\mathbf{x}_{1}\right)-H_{1 \mathrm{~B}}\left(\mathbf{x}_{2}\right)\right] \Gamma\left(\mathbf{x}_{1}, \mathbf{x}_{2}, t\right) \\
& +\left\{\int d \mathbf { y } V ( \mathbf { x } _ { 1 } - \mathbf { y } ) \left[\left\langle\psi^{\dagger}\left(\mathbf{x}_{2}\right) \psi^{\dagger}(\mathbf{y}) \psi(\mathbf{y}) \psi\left(\mathbf{x}_{1}\right)\right\rangle_{t}^{c}\right.\right. \\
& \quad+\Psi(\mathbf{y}, t)\left\langle\psi^{\dagger}\left(\mathbf{x}_{2}\right) \psi^{\dagger}(\mathbf{y}) \psi\left(\mathbf{x}_{1}\right)\right\rangle_{t}^{c} \\
& \quad+\Psi\left(\mathbf{x}_{1}, t\right)\left\langle\psi^{\dagger}\left(\mathbf{x}_{2}\right) \psi^{\dagger}(\mathbf{y}) \psi(\mathbf{y})\right\rangle_{t}^{c} \\
& \left.\quad+\Gamma\left(\mathbf{y}, \mathbf{x}_{2}, t\right) \Gamma\left(\mathbf{x}_{1}, \mathbf{y}, t\right)+\Gamma\left(\mathbf{x}_{1}, \mathbf{x}_{2}, t\right) \Gamma(\mathbf{y}, \mathbf{y}, t)\right] \\
& +\int d \mathbf{y} V\left(\mathbf{x}_{1}-\mathbf{y}\right) \Psi^{*}(\mathbf{y}, t)\left[\left\langle\psi^{\dagger}\left(\mathbf{x}_{2}\right) \psi(\mathbf{y}) \psi\left(\mathbf{x}_{1}\right)\right\rangle_{t}^{c}\right. \\
& \left.\quad+\Psi\left(\mathbf{x}_{1}, t\right) \Gamma\left(\mathbf{y}, \mathbf{x}_{2}, t\right)+\Psi(\mathbf{y}, t) \Gamma\left(\mathbf{x}_{1}, \mathbf{x}_{2}, t\right)\right] \\
& +\int d \mathbf{y} V\left(\mathbf{x}_{1}-\mathbf{y}\right) \Phi^{*}\left(\mathbf{y}, \mathbf{x}_{2}, t\right) \\
& \left.\quad \int\left[\Phi\left(\mathbf{x}_{1}, \mathbf{y}, t\right)+\Psi\left(\mathbf{x}_{1}, t\right) \Psi(\mathbf{y}, t)\right]\right\} \\
& \quad\left\{\mathbf{x}_{1} \leftrightarrow \mathbf{x}_{2}\right\}^{*}
\end{aligned}
$$

are obtained. Here $\left\{\mathbf{x}_{1} \leftrightarrow \mathbf{x}_{2}\right\}$ denotes the exchange of the coordinates $\mathbf{x}_{1}$ and $\mathbf{x}_{2}$ in the bracket and

$$
H_{2 \mathrm{~B}}\left(\mathbf{x}_{1}, \mathbf{x}_{2}\right)=H_{1 \mathrm{~B}}\left(\mathbf{x}_{1}\right)+H_{1 \mathrm{~B}}\left(\mathbf{x}_{2}\right)+V\left(\mathbf{x}_{1}-\mathbf{x}_{2}\right)
$$

denotes the two body Hamiltonian.

As proposed by Fricke [7], a first approach to close the dynamic equations for cumulants could consist in keeping all cumulants up to a certain order $n$. If, for example, $n=$ 1 is chosen Eq. (12) yields the Gross Pitaevskii equation in the Born approximation,

$$
\begin{aligned}
i \hbar \frac{\partial}{\partial t} \Psi(\mathbf{x}, t)= & {\left[H_{1 \mathrm{~B}}(\mathbf{x})+\int d \mathbf{y} V(\mathbf{x}-\mathbf{y})|\Psi(\mathbf{y}, t)|^{2}\right] } \\
& \times \Psi(\mathbf{x}, t) .
\end{aligned}
$$

Neglecting all cumulants containing three or four field operators in Eqs. (12), (13) and (14) yields a system of time dependent first order Hartree Fock Bogoliubov equations which constitute the second order approximation. An alternative derivation of these second order equations, in the contact potential approximation, which includes applications to the Timmermans model of molecular formation in condensates [9] can be found in Ref. [10]. An independent approach which contains also the cumulants up to the third order has been proposed by Proukakis and one of the authors [11].

These methods neglect multiple scattering in the equations for the last two relevant cumulants and can be interpreted as an expansion in terms of $V\left(t-t_{0}\right) / \hbar$, where $t-t_{0}$ is the time passed since the initial time. For this reason they are applicable mainly for the description of short time dynamics [7]. The multiple scattering contributions are contained in the dynamic equations for the cumulants of the order of $n+1, n+2$, etc..

A systematic extension of the previous methods, which accounts for multiple scattering in all equations of motion for the normal ordered cumulants up to the order of $n$, can be achieved by including the free dynamics of the normal ordered cumulants of the order of $n+1$ and $n+2$. Here, free dynamics means neglecting all products of normal ordered cumulants containing $n+3$ or $n+4$ field operators in the equations of motion for the normal ordered cumulants of the order of $n+1$ and $n+2$. As will be shown in Subsection IV A, including multiple scattering in all equations of motion at a given order of approximation significantly extends their range of validity.

\section{FIRST ORDER APPROACH}

In this section the extended cumulant method will be illustrated for the lowest order $n=1 \quad$ approximation. As in the case of the related Gross Pitaevskii approach, the resulting lowest order dynamic equation is applicable only as long as the influence of the non condensed fraction on the condensate can be neglected. This, in turn, restricts the initial states of the gas we can treat with this approximation.

Neglecting all products of cumulants containing four or five field operators in the equations of motion for the cumulants of the order of two and three, the dynamic equation for the condensate wave function is given by Eq. (12). According to Eqs. (13) and (14) the respective equations for $\Phi$ and $\Gamma$ in the same approximation become

$$
\begin{aligned}
i \hbar \frac{\partial}{\partial t} \Phi\left(\mathbf{x}_{1}, \mathbf{x}_{2}, t\right)= & H_{2 \mathrm{~B}}\left(\mathbf{x}_{1}, \mathbf{x}_{2}\right) \Phi\left(\mathbf{x}_{1}, \mathbf{x}_{2}, t\right) \\
& +V\left(\mathbf{x}_{1}-\mathbf{x}_{2}\right) \Psi\left(\mathbf{x}_{1}, t\right) \Psi\left(\mathbf{x}_{2}, t\right)
\end{aligned}
$$


and

$$
i \hbar \frac{\partial}{\partial t} \Gamma\left(\mathbf{x}_{1}, \mathbf{x}_{2}, t\right)=\left[H_{1 \mathrm{~B}}\left(\mathbf{x}_{1}\right)-H_{1 \mathrm{~B}}\left(\mathbf{x}_{2}\right)\right] \Gamma\left(\mathbf{x}_{1}, \mathbf{x}_{2}, t\right) .
$$

Among all third order normal ordered cumulants, only those which appear on the right hand side of Eq. (12) have to be considered. We first expand the dynamic equation for the correlation function $\left\langle\psi^{\dagger}\left(\mathbf{x}_{3}\right) \psi\left(\mathbf{x}_{2}\right) \psi\left(\mathbf{x}_{1}\right)\right\rangle_{t}$ according to Eq. (6) and neglect products of normal ordered cumulants containing five field operators. The equation of motion for the only relevant normal ordered third order cumulant is then given by:

$$
\begin{aligned}
& i \hbar \frac{\partial}{\partial t}\left\langle\psi^{\dagger}\left(\mathbf{x}_{3}\right) \psi\left(\mathbf{x}_{2}\right) \psi\left(\mathbf{x}_{1}\right)\right\rangle_{t}^{c} \\
& =\left[H_{2 \mathrm{~B}}\left(\mathbf{x}_{1}, \mathbf{x}_{2}\right)-H_{1 \mathrm{~B}}\left(\mathbf{x}_{3}\right)\right]\left\langle\psi^{\dagger}\left(\mathbf{x}_{3}\right) \psi\left(\mathbf{x}_{2}\right) \psi\left(\mathbf{x}_{1}\right)\right\rangle_{t}^{c} \\
& +V\left(\mathbf{x}_{1}-\mathbf{x}_{2}\right)[ \\
& \quad+\left(\mathbf{x}_{2}, t\right) \Gamma\left(\mathbf{x}_{1}, \mathbf{x}_{3}, t\right) \\
& \left.+\Psi\left(\mathbf{x}_{1}, t\right) \Gamma\left(\mathbf{x}_{2}, \mathbf{x}_{3}, t\right)\right] .
\end{aligned}
$$

Equations (17), (18) and (19) can be solved formally in terms of the time dependent two body Green's function. A detailed description of the Green's function methods applied in this article is given in Ref. [12]. The retarded two body Green's function is related to the respective time development operator $U_{2 \mathrm{~B}}\left(t, t_{0}\right)=\exp \left[-i H_{2 \mathrm{~B}}(t-\right.$ $\left.t_{0}\right) / \hbar$ ] through

$$
G_{2 \mathrm{~B}}^{(+)}\left(t, t_{0}\right)=\frac{1}{i \hbar} \theta\left(t-t_{0}\right) U_{2 \mathrm{~B}}\left(t, t_{0}\right),
$$

where $\theta\left(t-t_{0}\right)$ is the step function which yields unity for $t>t_{0}$ and vanishes elsewhere. Equation (20) shows that $G_{2 \mathrm{~B}}^{(+)}$obeys the equation:

$$
\left(i \hbar \frac{\partial}{\partial t}-H_{2 \mathrm{~B}}\right) G_{2 \mathrm{~B}}^{(+)}\left(t, t_{0}\right)=\delta\left(t-t_{0}\right) .
$$

A convenient representation of the formal solutions to Eqs. (17), (18) and (19) is achieved by changing from position space to the basis defined by the trap states $\phi_{i}$. In this representation the field operators $\psi$ become single mode annihilation operators $a_{i}=\int d \mathbf{x} \phi_{i}^{*}(\mathbf{x}) \psi(\mathbf{x})$, and the relevant cumulants are given by $\Psi_{i}(t)=\left\langle a_{i}\right\rangle_{t}^{c}$, $\Phi_{i j}(t)=\left\langle a_{j} a_{i}\right\rangle_{t}^{c}$ and $\Gamma_{i j}(t)=\left\langle a_{j}^{\dagger} a_{i}\right\rangle_{t}^{c}$. Using Eq. 21) the formal solution for the pair function in Eq. (17) can be written in the form:

$$
\begin{aligned}
& \Phi_{i j}(t)=\sum_{k_{1} k_{2}}{ }_{s}\left\langle i, j\left|U_{2 \mathrm{~B}}\left(t, t_{0}\right)\right| k_{1}, k_{2}\right\rangle_{s} \Phi_{k_{1} k_{2}}\left(t_{0}\right) \\
& +\sum_{k_{1} k_{2}} \int_{t_{0}}^{t} d t_{1 s}\left\langle i, j\left|G_{2 \mathrm{~B}}^{(+)}\left(t, t_{1}\right) V\right| k_{1}, k_{2}\right\rangle_{s} \Psi_{k_{1}}\left(t_{1}\right) \Psi_{k_{2}}\left(t_{1}\right) .
\end{aligned}
$$

Equation (18) yields the density matrix of the non condensed fraction thus:

$$
\Gamma_{i j}(t)=e^{-i\left(E_{i}-E_{j}\right)\left(t-t_{0}\right) / \hbar} \Gamma_{i j}\left(t_{0}\right) .
$$

In the same way, the formal solution for the third order cumulant in Eq. (19) becomes

$$
\begin{aligned}
\left\langle a_{k}^{\dagger} a_{j} a_{i}\right\rangle_{t}^{c}= & \sum_{k_{1} k_{2}}{ }_{s}\left\langle i, j\left|U_{2 \mathrm{~B}}\left(t, t_{0}\right)\right| k_{1}, k_{2}\right\rangle_{s} \\
& \times\left\langle a_{k}^{\dagger} a_{k_{2}} a_{k_{1}}\right\rangle_{t_{0}}^{c} e^{i E_{k}\left(t-t_{0}\right) / \hbar} \\
& +\sum_{k_{1} k_{2}} \int_{t_{0}}^{t} d t_{1 s}\left\langle i, j\left|G_{2 \mathrm{~B}}^{(+)}\left(t, t_{1}\right) V\right| k_{1}, k_{2}\right\rangle_{s} \\
& \times \Psi_{k_{1}}\left(t_{1}\right) \Gamma_{k_{2} k}\left(t_{1}\right) e^{i E_{k}\left(t-t_{1}\right) / \hbar} .
\end{aligned}
$$

Here $t_{0}$ denotes the initial time and $|i, j\rangle_{s}$ is the (not necessarily normalized) symmetrized product of trap states $\left[\phi_{i}\left(\mathbf{x}_{1}\right) \phi_{j}\left(\mathbf{x}_{2}\right)+\phi_{i}\left(\mathbf{x}_{2}\right) \phi_{j}\left(\mathbf{x}_{1}\right)\right] / 2$. According to Eqs. (23) and (24) in the first order cumulant approach, the density matrix of the non condensed fraction $\Gamma_{i j}(t)$ and the third order cumulant $\left\langle a_{k}^{\dagger} a_{j} a_{i}\right\rangle_{t}^{c}$ will not evolve in time as long as they vanish initially. Their contributions can therefore be neglected.

Equations (22), (23) and (24) inserted into Eq. (12) yield the closed nonlinear Schrödinger equation for the condensate wave function

$$
\begin{aligned}
& i \hbar \frac{\partial}{\partial t} \Psi_{i}(t)=E_{i} \Psi_{i}(t) \\
& \quad+\sum_{k_{1} k_{2} k_{3}} \int_{t_{0}}^{\infty} d t_{1 s}\left\langle i, k_{3}\left|T_{2 \mathrm{~B}}^{(+)}\left(t, t_{1}\right)\right| k_{1}, k_{2}\right\rangle_{s} \\
& \quad \times \Psi_{k_{1}}\left(t_{1}\right) \Psi_{k_{2}}\left(t_{1}\right) \Psi_{k_{3}}^{*}(t) .
\end{aligned}
$$

Here, $T_{2 \mathrm{~B}}^{(+)}$denotes the time dependent and retarded two body transition matrix

$$
T_{2 \mathrm{~B}}^{(+)}\left(t, t_{0}\right)=V \delta\left(t-t_{0}\right)+V G_{2 \mathrm{~B}}^{(+)}\left(t, t_{0}\right) V .
$$

The initial pair function $\Phi_{k_{1} k_{2}}\left(t_{0}\right)$, which accounts for collisions between condensate atoms that occur before $t_{0}$, is neglected in Eq. (25). If the condensate is prepared as an ideal gas ground state $\Phi_{k_{1} k_{2}}\left(t_{0}\right)$ vanishes exactly [13. . Recent experiments using magnetic fields to manipulate the pair interaction 14] have shown how to prepare such an initial state of a Bose condensed gas. In general, the state of the gas will include these initial correlations produced by collisions before the initial time we are considering.

It is worth noting that according to Eqs. (26) and (20) in the time integral on the right hand side of Eq. (25) the condensate wave function is evaluated only at the present time $t_{1}=t$ or in the past. This so called non-Markovian property of the nonlinear Schrödinger equation results from the influence of the higher order cumulants on the condensate, which modifies its dynamics.

\section{A. Equilibrium properties}

To describe many present day experiments we can use the Markov approximation in Eq. (25). In order to obtain 
the Markov limit, the condensate is assumed to be in an ideal gas initial state and the condensate wave function is transformed to the interaction picture

$$
\Psi_{i}(t)=\Psi_{i}^{\mathrm{I}}(t) e^{-i E_{i} t / \hbar} .
$$

For a dilute gas the non Markovian interaction term on the right hand side of Eq. (25) can be considered as a small perturbation of the first, i.e. ideal gas, contribution. This in turn leads to a weak time dependence of $\Psi_{i}^{\mathrm{I}}(t)$. In the interaction picture representation Eq. (25) reads

$$
\begin{aligned}
& i \hbar \frac{\partial}{\partial t} \Psi_{i}^{\mathrm{I}}(t)=\sum_{k_{1} k_{2} k_{3}} \int_{t_{0}}^{\infty} d t_{1 s}\left\langle i, k_{3}\left|T_{2 \mathrm{~B}}^{(+)}\left(t, t_{1}\right)\right| k_{1}, k_{2}\right\rangle_{s} \\
& \times e^{-i\left(E_{k_{1}}+E_{k_{2}}\right) t_{1} / \hbar} \Psi_{k_{1}}^{\mathrm{I}}\left(t_{1}\right) \Psi_{k_{2}}^{\mathrm{I}}\left(t_{1}\right)\left[\Psi_{k_{3}}^{\mathrm{I}}(t)\right]^{*} e^{i\left(E_{k_{3}}+E_{i}\right) t / \hbar} .
\end{aligned}
$$

According to Eq. (26) the time dependent transition matrix $T_{2 \mathrm{~B}}^{(+)}\left(t, t_{1}\right)$ is sharply peaked at $t_{1}=t$ with a width determined by the two body collisional duration. As $\Psi_{i}^{\mathrm{I}}(t)$ is slowly varying on this time scale, all interaction picture condensate wave functions can be evaluated at $t_{1}=t$ in Eq. (28). The remaining time integral in Eq. (28) then simply contains a Fourier transform of the time dependent transition matrix Eq. (26) and plays the role of a coupling function

$$
\begin{gathered}
\mathcal{T}_{2 \mathrm{~B}}\left(i, k_{3} ; k_{1}, k_{2}\right)=\int_{-\infty}^{t-t_{0}} d \tau_{s}\left\langle i, k_{3}\left|T_{2 \mathrm{~B}}^{(+)}(t, t-\tau)\right| k_{1}, k_{2}\right\rangle_{s} \\
\times e^{i\left(E_{k_{1}}+E_{k_{2}}\right) \tau / \hbar}
\end{gathered}
$$

Here, $\mathcal{T}_{2 \mathrm{~B}}$ becomes approximately independent of time as soon as $t-t_{0}$ exceeds the two body collisional duration. According to Eq. (26) the time dependent transition ma$\operatorname{trix} T_{2 \mathrm{~B}}^{(+)}$is related to the usual two body $T$ matrix 12 through

$$
T_{2 \mathrm{~B}}^{(+)}(t, t-\tau)=\frac{1}{2 \pi \hbar} \int d E e^{-i E \tau / \hbar} T_{2 \mathrm{~B}}(E+i 0),
$$

which still contains the trapping potential. Here " $i 0$ " denotes an imaginary energy " $i \varepsilon$ ", where the positive parameter $\varepsilon$ is taken to zero after performing the energy integral. In many present day experiments the trapping potential is nearly constant on the scale of the spatial range of the two body interaction potential, so the two body $T$ matrix in Eq. (30) can be replaced by its free space counterpart $T_{2 \mathrm{~B}}^{\text {free }}$. The time limit $t-t_{0} \rightarrow \infty$ in Eq. (29) then yields

$$
\mathcal{T}_{2 \mathrm{~B}}\left(i, k_{3} ; k_{1}, k_{2}\right)={ }_{s}\left\langle i, k_{3}\left|T_{2 \mathrm{~B}}^{\text {free }}\left(E_{k_{1}}+E_{k_{2}}+i 0\right)\right| k_{1}, k_{2}\right\rangle_{s} .
$$

As a consequence, the condensate wave function $\Psi_{i}(t)$ Eq. (28) obeys the following form of the Gross Pitaevskii equation:

$$
\begin{aligned}
& i \hbar \frac{\partial}{\partial t} \Psi_{i}(t)=E_{i} \Psi_{i}(t) \\
& \quad+\sum_{k_{1} k_{2} k_{3}} \mathcal{T}_{2 \mathrm{~B}}\left(i, k_{3} ; k_{1}, k_{2}\right) \Psi_{k_{1}}(t) \Psi_{k_{2}}(t) \Psi_{k_{3}}^{*}(t) .
\end{aligned}
$$

The coupling function in Eq. (31), however, exhibits an imaginary part which describes collisional losses of energetically excited condensate atoms. The physical significance of this imaginary part will be explained in Subsection IV B.

Close to equilibrium, the energy of occupied modes can be considered to be so small that the coupling function is purely real to a good approximation. The two body $T$ matrix can then be evaluated by means of the contact potential approximation

$$
\begin{aligned}
T_{2 \mathrm{~B}}^{\mathrm{free}}= & \int d \mathbf{p}_{1} d \mathbf{p}_{2} d \mathbf{p}_{3} d \mathbf{p}_{4} \delta\left(\mathbf{p}_{4}+\mathbf{p}_{3}-\mathbf{p}_{1}-\mathbf{p}_{2}\right) \\
& \times\left|\mathbf{p}_{4}\right\rangle\left|\mathbf{p}_{3}\right\rangle \frac{4 \pi \hbar^{2}}{m} a_{0}\left\langle\mathbf{p}_{1}\right|\left\langle\mathbf{p}_{2}\right|
\end{aligned}
$$

Here $\left|\mathbf{p}_{i}\right\rangle$ denotes a one body plane wave momentum state and $m$ the atomic mass while $a_{0}$ is the two body $s$ wave scattering length. In the contact potential approximation Eq. (32) can be shown to be formally equivalent to the Gross Pitaevskii equation in the Born approximation (see Eq. (16)) with the two body potential $V(\mathbf{r})$ replaced by $4 \pi \hbar^{2} a_{0} \delta(\mathbf{r}) / m$. Hence, in the position representation, the equilibrium limit of the Markovian nonlinear Schrödinger equation in Eq. (32) has the form:

$$
i \hbar \frac{\partial}{\partial t} \Psi(\mathbf{x}, t)=\left[H_{1 \mathrm{~B}}(\mathbf{x})+\frac{4 \pi \hbar^{2}}{m} a_{0}|\Psi(\mathbf{x}, t)|^{2}\right] \Psi(\mathbf{x}, t)
$$

This Gross Pitaevskii equation is transformed to its time independent form by means of the ansatz $\Psi(\mathbf{x}, t)=$ $\Psi(\mathbf{x}) \exp (-i \mu t / \hbar)$, where the energy parameter $\mu$ is usually termed the chemical potential. The Markov approximation of the first order cumulant approach thus leads to the correct Gross Pitaevskii equation, which describes virtually all equilibrium phenomena in present day experiments with Bose condensates near zero temperature.

\section{B. Scattering of two condensates}

In this subsection, the cumulant approach will be applied to describe the scattering of two condensates: a clear and important non-equilibrium case. In a typical experiment the different condensates are generated from a trapped parent condensate, at nearly zero temperature, by means of an optical standing wave pulse. The trap in some experiments can be switched off directly after the application of the light pulse at time $t_{0}$. The photon momentum $\mathbf{p}_{\mathrm{ph}}$ can be transferred to the atoms and is assumed to be much larger than the momentum spread in the parent condensate and also the momentum $m c$ associated with the speed of sound $c$. This implies that the condensate wave function prepared in this way contains three well separated momentum components with the respective central atomic momenta $\mathbf{p}= \pm 2 \mathbf{p}_{\mathrm{ph}}$ and $\mathbf{p}=0$. For simplicity, the following analysis is restricted 
to the presence of two distinct components. The condensate wave function can then be represented in the center of mass frame as 15

$$
\begin{aligned}
\Psi\left(\mathbf{x}, t_{0}\right)= & {\left[\Psi_{+}\left(\mathbf{x}, t_{0}\right) e^{i \mathbf{p}_{\mathrm{ph}} \cdot \mathbf{x} / \hbar}+\Psi_{-}\left(\mathbf{x}, t_{0}\right) e^{-i \mathbf{p}_{\mathrm{ph}} \cdot \mathbf{x} / \hbar}\right] } \\
& \times e^{-i \frac{\mathbf{p}_{\mathrm{ph}}^{2}}{2 m} t_{0} / \hbar} .
\end{aligned}
$$

Here the wave functions

$$
\Psi_{+}\left(\mathbf{x}, t_{0}\right)=A_{+} \Psi(\mathbf{x})
$$

and

$$
\Psi_{-}\left(\mathbf{x}, t_{0}\right)=A_{-} \Psi(\mathbf{x})
$$

are multiples of the stationary wave function of the parent condensate $\Psi(\mathbf{x})$. The latter is a solution of the time independent Gross Pitaevskii equation containing the trapping potential. The amplitudes $A_{+}$and $A_{-}$are normalized as $\left|A_{+}\right|^{2}+\left|A_{-}\right|^{2}=1$. This ensures that $\int d \mathbf{x}\left|\Psi\left(\mathbf{x}, t_{0}\right)\right|^{2}$ equals the number of atoms in the parent condensate, as long as the overlap in momentum space of the two components on the right hand side of Eq. (35) is negligible.

The following analysis of the scattering process is based on our non-Markovian nonlinear Schrödinger equation given by Eq. (25). In position space this equation has the following form:

$$
\begin{aligned}
& i \hbar \frac{\partial}{\partial t} \Psi(\mathbf{x}, t)=H_{1 \mathrm{~B}}(\mathbf{x}) \Psi(\mathbf{x}, t) \\
& +\int d \mathbf{y}_{1} d \mathbf{y}_{2} d \mathbf{y}_{3} \int_{t_{0}}^{\infty} d t_{1}\left\langle\mathbf{x}, \mathbf{y}_{3}\left|T_{2 \mathrm{~B}}^{(+)}\left(t, t_{1}\right)\right| \mathbf{y}_{1}, \mathbf{y}_{2}\right\rangle \\
& \quad \times \Psi\left(\mathbf{y}_{1}, t_{1}\right) \Psi\left(\mathbf{y}_{2}, t_{1}\right) \Psi^{*}\left(\mathbf{y}_{3}, t\right) .
\end{aligned}
$$

The initial condition Eq. (35) implies that the representation

$$
\begin{aligned}
\Psi(\mathbf{x}, t)= & {\left[\Psi_{+}(\mathbf{x}, t) e^{i \mathbf{p}_{\mathrm{ph}} \cdot \mathbf{x} / \hbar}+\Psi_{-}(\mathbf{x}, t) e^{-i \mathbf{p}_{\mathrm{ph}} \cdot \mathbf{x} / \hbar}\right] } \\
& \times e^{-i \frac{\mathbf{p}_{\mathrm{ph}}^{2}}{2 m} t / \hbar}
\end{aligned}
$$

provides a suitable interaction picture, where $\Psi_{+}(\mathbf{x}, t)$ and $\Psi_{-}(\mathbf{x}, t)$ are assumed to be slowly varying in space and time. This interaction picture representation was proposed by Band et al. in Ref. [15 and is equivalent to the slowly varying envelope approximation SVEA of nonlinear optics 16 . Equation (39) inserted into Eq. (38) yields the following equation of motion:

$$
\begin{aligned}
& \left\{e^{i \mathbf{p}_{\mathrm{ph}} \cdot \mathbf{x} / \hbar}\left[i \hbar\left(\frac{\partial}{\partial t}+\frac{\mathbf{p}_{\mathrm{ph}}}{m} \cdot \nabla \mathbf{x}\right)+\frac{\hbar^{2}}{2 m} \Delta_{\mathbf{x}}\right] \Psi_{+}(\mathbf{x}, t)\right\} \\
& +\left\{\mathbf{p}_{\mathrm{ph}} \leftrightarrow-\mathbf{p}_{\mathrm{ph}}\right\} \\
& =\int d \mathbf{y}_{1} d \mathbf{y}_{2} d \mathbf{y}_{3} \int_{t_{0}}^{\infty} d t_{1} e^{i \frac{\mathbf{p}_{\mathrm{ph}}^{2}}{m}\left(t-t_{1}\right) / \hbar} \\
& \quad \times\left\langle\mathbf{x}, \mathbf{y}_{3}\left|T_{2 \mathrm{~B}}^{(+)}\left(t, t_{1}\right)\right| \mathbf{y}_{1}, \mathbf{y}_{2}\right\rangle \\
& \quad \times\left[e^{i \mathbf{p}_{\mathrm{ph}} \cdot \mathbf{y}_{1} / \hbar} \Psi_{+}\left(\mathbf{y}_{1}, t_{1}\right)+e^{-i \mathbf{p}_{\mathrm{ph}} \cdot \mathbf{y}_{1} / \hbar} \Psi_{-}\left(\mathbf{y}_{1}, t_{1}\right)\right] \\
& \quad \times\left[e^{i \mathbf{p}_{\mathrm{ph}} \cdot \mathbf{y}_{2} / \hbar} \Psi_{+}\left(\mathbf{y}_{2}, t_{1}\right)+e^{-i \mathbf{p}_{\mathrm{ph}} \cdot \mathbf{y}_{2} / \hbar} \Psi_{-}\left(\mathbf{y}_{2}, t_{1}\right)\right] \\
& \quad \times\left[e^{-i \mathbf{p}_{\mathrm{ph}} \cdot \mathbf{y}_{3} / \hbar} \Psi_{+}^{*}\left(\mathbf{y}_{3}, t\right)+e^{i \mathbf{p}_{\mathrm{ph}} \cdot \mathbf{y}_{3} / \hbar} \Psi_{-}^{*}\left(\mathbf{y}_{3}, t\right)\right] .
\end{aligned}
$$

Here, $T_{2 \mathrm{~B}}^{(+)}\left(t, t_{1}\right)$ is the time dependent transition operator Eq. 26) in free space and $\left\{\mathbf{p}_{\mathrm{ph}} \leftrightarrow-\mathbf{p}_{\mathrm{ph}}\right\}$ means the exchange of $\mathbf{p}_{\mathrm{ph}}$ and $-\mathbf{p}_{\mathrm{ph}}$ and of $\Psi_{+}$and $\Psi_{-}$inside the bracket. The interaction picture wave functions $\Psi_{ \pm}\left(\mathbf{y}_{1}, t_{1}\right)$ and $\Psi_{ \pm}\left(\mathbf{y}_{2}, t_{1}\right)$ are slowly varying on the time scale of the two body collisional duration and can be evaluated at $t_{1}=t$ on the right hand side of Eq. (40). This leads to the Markov approximation. In virtue of Eq. (30) the remaining time integral yields

$$
\int_{t_{0}}^{\infty} d t_{1} e^{i \frac{\mathbf{p}_{\mathrm{ph}}^{2}}{m}\left(t-t_{1}\right) / \hbar} T_{2 \mathrm{~B}}^{(+)}\left(t, t_{1}\right)=T_{2 \mathrm{~B}}\left(\mathbf{p}_{\mathrm{ph}}^{2} / m+i 0\right)
$$

as soon as $t-t_{0}$ exceeds the two body collisional duration.

To perform the spatial integrations on the right hand side of Eq. (40) the position representation of the $T$ matrix in Eq. (41) is transformed to the momentum space and becomes

$$
\begin{aligned}
& \left\langle\mathbf{x}, \mathbf{y}_{3}\left|T_{2 \mathrm{~B}}\left(\mathbf{p}_{\mathrm{ph}}^{2} / m+i 0\right)\right| \mathbf{y}_{1}, \mathbf{y}_{2}\right\rangle \\
& =\frac{1}{(2 \pi \hbar)^{6}} \int d \mathbf{p}_{1} d \mathbf{p}_{2} d \mathbf{p}_{3} d \mathbf{p}_{4} \delta\left(\mathbf{p}_{1}+\mathbf{p}_{2}-\mathbf{p}_{3}-\mathbf{p}_{4}\right) \\
& \times\left\langle\frac{\mathbf{p}_{3}-\mathbf{p}_{4}}{2}\left|\hat{T}_{2 \mathrm{~B}}\left(\frac{\mathbf{p}_{\mathbf{p h}}^{2}}{m}-\frac{\left(\mathbf{p}_{1}+\mathbf{p}_{2}\right)^{2}}{4 m}+i 0\right)\right| \frac{\mathbf{p}_{1}-\mathbf{p}_{2}}{2}\right\rangle \\
& \times e^{i\left(\mathbf{p}_{4} \cdot \mathbf{x}+\mathbf{p}_{3} \cdot \mathbf{y}_{3}\right) / \hbar} e^{-i\left(\mathbf{p}_{1} \cdot \mathbf{y}_{1}+\mathbf{p}_{2} \cdot \mathbf{y}_{2}\right) / \hbar},
\end{aligned}
$$

where $\hat{T}_{2 \mathrm{~B}}$ is the $T$ matrix which contains only the relative motion of pairs of atoms. The momentum conserving $\delta$ function stems from the translational invariance of $T_{2 \mathrm{~B}}$ in the center of mass coordinates. For the experimentally relevant photon momenta $\mathbf{p}_{\mathrm{ph}}$, the $T$ matrix on the right hand side of Eq. (42) depends significantly on the related kinetic energy $\mathbf{p}_{\mathrm{ph}}^{2} / m$ and the contact potential approximation Eq. (33) cannot be used. In the Markov 
approximation Eq. (40) then reads

$$
\begin{aligned}
& \left\{e^{i \mathbf{p}_{\mathrm{ph}} \cdot \mathbf{x} / \hbar}\left[i \hbar\left(\frac{\partial}{\partial t}+\frac{\mathbf{p}_{\mathrm{ph}}}{m} \cdot \nabla \mathbf{x}\right)+\frac{\hbar^{2}}{2 m} \Delta_{\mathbf{x}}\right] \Psi_{+}(\mathbf{x}, t)\right\} \\
& +\left\{\mathbf{p}_{\mathrm{ph}} \leftrightarrow-\mathbf{p}_{\mathrm{ph}}\right\} \\
& =\frac{1}{\sqrt{2 \pi \hbar}} \int d \mathbf{p}_{1} d \mathbf{p}_{2} d \mathbf{p}_{3} d \mathbf{p}_{4} \delta\left(\mathbf{p}_{1}+\mathbf{p}_{2}-\mathbf{p}_{3}-\mathbf{p}_{4}\right) \\
& \times\left\langle\frac{\mathbf{p}_{3}-\mathbf{p}_{4}}{2}\left|\hat{T}_{2 \mathrm{~B}}\left(\frac{\mathbf{p}_{\mathrm{ph}}^{2}}{m}-\frac{\left(\mathbf{p}_{1}+\mathbf{p}_{2}\right)^{2}}{4 m}+i 0\right)\right| \frac{\mathbf{p}_{1}-\mathbf{p}_{2}}{2}\right\rangle \\
& \times\left\{\left[\Psi_{+}\left(\mathbf{p}_{1}-\mathbf{p}_{\mathrm{ph}}, t\right) \Psi_{+}\left(\mathbf{p}_{2}-\mathbf{p}_{\mathrm{ph}}, t\right) \Psi_{+}^{*}\left(\mathbf{p}_{3}-\mathbf{p}_{\mathrm{ph}}, t\right)\right.\right. \\
& \quad+\Psi_{+}\left(\mathbf{p}_{1}-\mathbf{p}_{\mathrm{ph}}, t\right) \Psi_{-}\left(\mathbf{p}_{2}+\mathbf{p}_{\mathrm{ph}}, t\right) \Psi_{+}^{*}\left(\mathbf{p}_{3}-\mathbf{p}_{\mathrm{ph}}, t\right) \\
& \quad+\Psi_{-}\left(\mathbf{p}_{1}+\mathbf{p}_{\mathrm{ph}}, t\right) \Psi_{+}\left(\mathbf{p}_{2}-\mathbf{p}_{\mathrm{ph}}, t\right) \Psi_{+}^{*}\left(\mathbf{p}_{3}-\mathbf{p}_{\mathrm{ph}}, t\right) \\
& \left.\quad+\Psi_{+}\left(\mathbf{p}_{1}-\mathbf{p}_{\mathrm{ph}}, t\right) \Psi_{+}\left(\mathbf{p}_{2}-\mathbf{p}_{\mathrm{ph}}, t\right) \Psi_{-}^{*}\left(\mathbf{p}_{3}+\mathbf{p}_{\mathrm{ph}}, t\right)\right] \\
& \left.\quad+\left[\mathbf{p}_{\mathrm{ph}} \leftrightarrow-\mathbf{p}_{\mathrm{ph}}\right]\right\} e^{i \mathbf{p}_{4} \cdot \mathbf{x} / \hbar},
\end{aligned}
$$

where $\Psi_{ \pm}(\mathbf{p}, t)$ denotes the Fourier transform

$$
\Psi_{ \pm}(\mathbf{p}, t)=\frac{1}{\sqrt{2 \pi \hbar}^{3}} \int d \mathbf{x} e^{-i \mathbf{p} \cdot \mathbf{x} / \hbar} \Psi_{ \pm}(\mathbf{x}, t) .
$$

The wave functions $\Psi_{ \pm}(\mathbf{x}, t)$ are slowly varying in space, and so their Fourier transform $\Psi_{ \pm}(\mathbf{p}, t)$ is sharply peaked at zero momentum $(\mathbf{p}=0)$ with a width determined by the initial momentum spread of the parent condensate. According to Eq. (30), the two body $T$ matrix $\hat{T}_{2 B}(E+i 0)$ is slowly varying on the scale of the energies corresponding to the inverse two body collisional duration. Its plane wave basis matrix elements $\left\langle\mathbf{p}_{\text {out }}\left|\hat{T}_{2 B}\right| \mathbf{p}_{\text {in }}\right\rangle$ are slowly varying in $\mathbf{p}_{\text {in }}$ and $\mathbf{p}_{\text {out }}$, on the scale of the momentum associated with the inverse range of the two body interaction potential $V(\mathbf{r})$. This can be seen through the relation:

$$
\begin{aligned}
\left\langle\mathbf{r}_{\text {out }}\left|\hat{T}_{2 \mathrm{~B}}\right| \mathbf{r}_{\text {in }}\right\rangle= & V\left(\mathbf{r}_{\text {in }}\right) \delta\left(\mathbf{r}_{\text {in }}-\mathbf{r}_{\text {out }}\right) \\
& +V\left(\mathbf{r}_{\text {out }}\right)\left\langle\mathbf{r}_{\text {out }}\left|\hat{G}_{2 \mathrm{~B}}\right| \mathbf{r}_{\text {in }}\right\rangle V\left(\mathbf{r}_{\text {in }}\right)
\end{aligned}
$$

where $\hat{G}_{2 \mathrm{~B}}$ denotes the two body energy-dependent Green's function for the relative motion only. As a consequence, the plane wave matrix element of the $T$ matrix $\hat{T}_{2 B}$ on the right hand side of Eq. (43) can be evaluated at the respective momenta determined by the products of momentum space condensate wave functions and by the momentum conserving $\delta$ function. Performing the momentum space integrals then transforms the condensate wave functions $\Psi_{ \pm}$back to position space and leads to the following equation of motion:

$$
\begin{aligned}
& \left\{e^{i \mathbf{p}_{\mathrm{ph}} \cdot \mathbf{x} / \hbar}\left[i \hbar\left(\frac{\partial}{\partial t}+\frac{\mathbf{p}_{\mathrm{ph}}}{m} \cdot \nabla \mathbf{x}\right)+\frac{\hbar^{2}}{2 m} \Delta_{\mathbf{x}}\right] \Psi_{+}(\mathbf{x}, t)\right\} \\
& +\left\{\mathbf{p}_{\mathrm{ph}} \leftrightarrow-\mathbf{p}_{\mathrm{ph}}\right\} \\
& =\left\{e ^ { i \mathbf { p } _ { \mathrm { ph } } \cdot \mathbf { x } / \hbar } \frac { 4 \pi \hbar ^ { 2 } } { m } \left[a_{0}\left|\Psi_{+}(\mathbf{x}, t)\right|^{2} \Psi_{+}(\mathbf{x}, t)\right.\right. \\
& \left.\quad-\left[f\left(\mathbf{k}_{\mathrm{ph}}, \mathbf{k}_{\mathrm{ph}}\right)+f\left(\mathbf{k}_{\mathrm{ph}},-\mathbf{k}_{\mathrm{ph}}\right)\right]\left|\Psi_{-}(\mathbf{x}, t)\right|^{2} \Psi_{+}(\mathbf{x}, t)\right] \\
& \left.+e^{3 i \mathbf{p}_{\mathrm{ph}} \cdot \mathbf{x} / \hbar}\left\langle-2 \mathbf{p}_{\mathrm{ph}}\left|\hat{T}_{2 \mathrm{~B}}(i 0)\right| 0\right\rangle \Psi_{-}^{*}(\mathbf{x}, t) \Psi_{+}^{2}(\mathbf{x}, t)\right\} \\
& +\left\{\mathbf{p}_{\mathrm{ph}} \leftrightarrow-\mathbf{p}_{\mathrm{ph}}\right\} .
\end{aligned}
$$

Here, $\mathbf{k}_{\mathrm{ph}}=\mathbf{p}_{\mathrm{ph}} / \hbar$ denotes the wave vector associated with the photon momentum $\mathbf{p}_{\mathrm{ph}}$ and

$$
\begin{aligned}
f\left(\mathbf{k}_{\text {out }}, \mathbf{k}_{\text {in }}\right)= & -2 \pi^{2} m \hbar \\
& \times\left\langle\hbar \mathbf{k}_{\text {out }}\left|\hat{T}_{2 \mathrm{~B}}\left(\hbar^{2} k^{2} / m+i 0\right)\right| \hbar \mathbf{k}_{\text {in }}\right\rangle
\end{aligned}
$$

is the scattering amplitude, where the wave vectors $\mathbf{k}_{\text {in }}$ and $\mathbf{k}_{\text {out }}$ are on the energy shell $\left|\mathbf{k}_{\text {out }}\right|=\left|\mathbf{k}_{\text {in }}\right|=k$. Equation (46) contains two well separated momentum components on its left hand side and four on the right hand side. Keeping only the phase matched terms yields a system of two coupled nonlinear Schrödinger equations. These are given by

$$
\begin{aligned}
& {\left[i \hbar\left(\frac{\partial}{\partial t}+\hbar \frac{\mathbf{k}_{\mathrm{ph}}}{m} \cdot \nabla \mathbf{x}\right)+\frac{\hbar^{2}}{2 m} \Delta_{\mathbf{x}}\right.} \\
& \left.-\frac{4 \pi \hbar^{2}}{m} a_{0}\left|\Psi_{+}(\mathbf{x}, t)\right|^{2}\right] \Psi_{+}(\mathbf{x}, t) \\
& =-\frac{4 \pi \hbar^{2}}{m}\left[f\left(\mathbf{k}_{\mathrm{ph}}, \mathbf{k}_{\mathrm{ph}}\right)+f\left(\mathbf{k}_{\mathrm{ph}},-\mathbf{k}_{\mathrm{ph}}\right)\right] \\
& \quad \times\left|\Psi_{-}(\mathbf{x}, t)\right|^{2} \Psi_{+}(\mathbf{x}, t)
\end{aligned}
$$

for the wave function $\Psi_{+}$and the analogous relation for $\Psi_{-}$where $\mathbf{k}_{\mathrm{ph}}$ and $-\mathbf{k}_{\mathrm{ph}}$ as well as $\Psi_{+}$and $\Psi_{-}$are exchanged.

As a result of the Markov approximation the $T$ matrix elements in Eq. (48) are evaluated on the two-body energy shell and can be represented in terms of the scattering amplitude Eq. (47). The experimentally relevant photon momenta are sufficiently small for the scattering amplitudes on the right hand side of Eq. (48) to be in the isotropic limit. According to effective range theory 12. $f\left(\mathbf{k}_{\text {out }}, \mathbf{k}_{\text {in }}\right)$ in Eq. (47) can then be expanded in the form:

$$
f\left(\mathbf{k}_{\text {out }}, \mathbf{k}_{\text {in }}\right)=-a_{0}+i a_{0}\left(k a_{0}\right)+\mathcal{O}\left(k^{2}\right) .
$$

Equation 49) inserted into Eq. (48) recovers the result of Band et al. in Ref. [15]. In the latter article, the two body energy conservation in the collisions was assumed for physical reasons and the imaginary part on the right 
hand side of Eq. (49) included, on an heuristic basis in the Gross Pitaevskii equation, in the form of Eq. (34). The imaginary correction term includes the loss of those atoms from the condensates that are elastically scattered out of the forward direction. As reported in Ref. [15], these losses have been observed experimentally in four wave mixing experiments with Bose condensates [1] and exhibit an excellent agreement with the predictions of the appropriate generalization of Eq. (48).

\section{CONCLUSIONS}

We have shown in this paper how we may systematically obtain a theoretical description of the dynamics of dilute Bose gases which are far from their thermal equilibrium. The underlying cumulant approach is based on truncating the infinite hierarchy of quantum evolution equations for correlation functions of boson field operators in accordance with Wick's theorem of statistical mechanics. We extend this approach by giving a general scheme for including multiple scattering in all dynamic equations. This extended approach describes the inter-particle collisions in terms of scattering amplitudes rather than bare potentials.

The lowest order approximation consists in a non Markovian nonlinear Schrödinger equation. When applied to initial conditions close to the thermal equilibrium at zero temperature our lowest order approach recovers the time dependent Gross Pitaevskii equation. Applied to the scattering of two condensates at a sharply defined relative velocity, which is high in comparison with the internal velocity spread in each condensate, we have shown that the non Markovian nonlinear Schrödinger equation goes beyond the Gross Pitaevskii approach and describes the experimentally observed collisional loss of condensate atoms. Hence, including multiple scattering not simply justifies the common contact potential approximation but accounts for important new physical phenomena.

The second order basic cumulant approach in the contact potential approximation was shown to be equivalent to the first order time dependent Hartree-Fock Bogoliubov theory derived in Ref. [10]. A detailed description of the physical significance of the corresponding extended approach, which includes multiple scattering, goes beyond the scope of this article and will appear elsewhere [17.

Our first order approach should also be applicable when the relative bulk motion is still rapid but its velocity is not sharply defined. This situation occurs during the collapse of a Bose condensed gas with a negative $s$ wave scattering length. Very recently, the dynamics of a collapse and the related losses of condensate atoms have been determined experimentally in Ref. [3].

\section{ACKNOWLEDGMENTS}

We would like to gratefully acknowledge inspiring discussions with Paul Julienne. This work was supported by the Alexander von Humboldt Foundation and the United Kingdom Engineering and Physical Sciences Research Council and the European Union.

[1] L. Deng, E. W. Hagley, J. Wen, M. Trippenbach, Y. Band, P. S. Julienne, J. E. Simsarian, K. Helmerson, S. L. Rolston, and W. D. Phillips, Nature (London) 398, 218-220 (1999).

[2] J. L. Roberts, N. R. Claussen, S. L. Cornish, E. A. Donley, E. A. Cornell and C. E. Wieman, Phys. Rev Lett. 86, 4211 (2001).

[3] E. A. Donley, N. R. Claussen, S. L. Cornish, J. L. Roberts, E. A. Cornell and C. E. Wieman, Nature (London) 412, 295 (2001).

[4] C. W. Gardiner and P. Zoller, Phys. Rev. A 61, 033601 (2000) and references therein.

[5] M. Holland, J. Williams, and J. Cooper, Phys. Rev. A 55, 3670 (1997).

[6] R. Walser, J. Williams, J. Cooper, and M. Holland, Phys. Rev. A 59, 3878 (1999).

[7] J. Fricke, Ann. Phys. (N.Y.) 252, 479 (1996).

[8] A. L. Fetter, J. D. Walecka, Quantum Theory of ManyParticle Systems, (McGraw-Hill, New York, 1971).

[9] E. Timmermans, P. Tommasini, R. Côté, M. Hussein, and A. Kerman, Phys. Rev. Lett. 83, 2691 (1999).

[10] M. Holland, J. Park and R. Walser, Phys. Rev. Lett. 86, 1915 (2001).

[11] N. P. Proukakis and K. Burnett, J. Res. Natl. Inst. Stand. Technol. 101, 457 (1996).

[12] R. G. Newton, Scattering Theory of Waves and Particles (Springer, New York, 1982).

[13] The density matrix of a purely condensed ideal gas is given by a pure coherent state of the lowest trap mode. With the latter chosen as the initial state of the interacting dilute gas all initial normal ordered correlation functions factorize into products of the condensate wave function or its conjugate, and Eq. (6) shows recursively that all respective cumulants except the condensate wave function vanish.

[14] S. L. Cornish, N. R. Claussen, J. L. Roberts, E. A. Cornell, and C. E. Wieman, Phys. Rev. Lett. 85, 1795 (2000).

[15] Y. B. Band, M. Trippenbach, J. P. Burke, and P. S. Julienne, Phys. Rev. Lett. 84, 5462 (2000).

[16] See, e.g., E. M. Dianov, A. B. Grudinin, A. M. Prokhorov and V. N. Serkin in Optical Solitons - Theory and Experiment, edited by J. R. Taylor (Cambridge University Press, Cambridge, 1992).

[17] T. Köhler and K. Burnett (unpublished). 